\title{
Assessment of a Medication Deprescribing Tool on Polypharmacy and Cost Avoidance
}

\author{
Janie K. Constantino-Corpuz, PharmD; and Monika Tara D. Alonso, PharmD, BCGP
}

\begin{abstract}
Purpose: The number of patients who take 5 or more medications-polypharmacy-is increasing. Patients with polypharmacy are at risk for adverse drug events, drugdrug interactions, geriatric-related syndromes, falls, and hospitalizations.
\end{abstract}

Methods: A retrospective chart review was performed to assess the impact of the VIONE deprescribing tool on polypharmacy and cost avoidance when used by primary care physicians and pharmacists in patient aligned care team primary care clinics at Veteran Affairs Southern Nevada Healthcare System in North Las Vegas. The efficacy of the tool was determined by calculating the total amount of medications deprescribed as well as the estimated annualized costs avoided for the health care system.

Results: In a 3-month period, 1060 medications were deprescribed. The total estimated cost avoidance for the facility was $\$ 84,030.46$.

Conclusion: The VIONE tool may be useful in improving patient safety through deprescribing and discontinuing potentially inappropriate medications. Use of this deprescribing tool also may reduce the cost of care associated with overprescribing.

\begin{abstract}
Janie Constantino-Corpuz is a Patient Aligned Care Team Clinical Pharmacist, and Monika Alonso is a Home-Based Primary Care Clinical Pharmacist, both at Veteran Affairs

Southern Nevada Healthcare System in North Las Vegas. Correspondence:

Janie Constantino-Corpuz (janie.constantino-corpuz@ va.gov)
\end{abstract}

Fed Pract. 2021;38(7) Published online July 15. doi:10.12788/fp.0146
A ccording to the Centers for Disease Control and Prevention National Center for Health Statistics (NCHS), the use of prescription drugs has increased in the past half century. Although prescription drugs have played an important role in preventing, controlling, and delaying onset or progression of disease, their growth in use also has posed many risks. ${ }^{1}$ One ramification of this growth is the occurrence of polypharmacy, which does not have a universal, clear definition. In general, it can be described as the concurrent use of multiple medications by a single patient to treat one or more medical ailments. Five or more medications taken simultaneously is the most common definition to date, but this is just one of many acceptable definitions and that varies from one health care facility to another. ${ }^{1,2}$

Regardless of the cutoffs established to indicate polypharmacy, its incidence can result in poor and potentially harmful health outcomes. Polypharmacy increases the risk of experiencing adverse drug events (ADEs), drug-drug interactions (DDIs), geriatric-related syndromes, falls, hospitalization, and mortality. Issues with adherence may begin to unfold secondary to increased pill burden. Both the patient and the health care system may encounter financial strain, as polypharmacy can lead to unnecessary and essentially preventable costs of care. When evaluating the likelihood of polypharmacy based on age group, NCHS found that $47.5 \%$ of patients taking $\geq 5$ medications were aged $\geq 65$ years. ${ }^{1-5}$ This indicates that polypharmacy is of great concern in the geriatric population, which also represents a large proportion of individuals accessing Veterans Health Administration (VHA) care.

\section{DEPRESCRIBING}

Deprescribing is the act of withdrawing or discontinuing potentially inappropriate medications (PIM), or medications used by older patients harboring ADEs that generally outweigh the clinical benefits of the drug. Deprescribing is an effective tool for managing or reducing polypharmacy. A variety of tools have been created whose sole purpose is to simplify deprescribing. Some tools explicitly identify PIM and are widely familiar in medical practice. Examples are the Beers Criteria developed in 1991 or Screening Tool to Alert Right Treatment/ Screening Tool of Older Persons Prescriptions (START/STOPP) criteria created in 2003. Other tools that are less commonplace but equally as resourceful are MedStopper and Deprescribing.org. The former was launched in 2015 and is a Canadian online system that provides risk assessments for medications with guidance for tapering or stopping medications if continuation of the drug presents higher risk than benefit. ${ }^{5-7}$ The latter is a full-fledged website developed by a physician, a pharmacist, and their research teams that serves as an exchange hub for deprescribing information. 
TABLE 1 VIONE Mnemonic

\begin{tabular}{|c|c|c|c|}
\hline & Descriptions & Suggested Actions & Examples \\
\hline V & Vital, life sustaining & Continue; monitor dosage, frequency, and adjust accordingly & Insulin for diabetes mellitus \\
\hline I & $\begin{array}{l}\text { Important for quality of life, } \\
\text { although not life sustaining }\end{array}$ & $\begin{array}{l}\text { Continue/consolidate medication; use the prescription with } \\
\text { the least adverse effects and most cost-effective }\end{array}$ & Pain medication; stool softener \\
\hline $\mathrm{O}$ & Optional & $\begin{array}{l}\text { Weigh benefits vs risks of medication; seriously consider } \\
\text { reducing or stopping }\end{array}$ & $\begin{array}{l}\text { Diabetes mellitus test strips and insulin } \\
\text { for actively dying patient }\end{array}$ \\
\hline $\mathrm{N}$ & $\begin{array}{l}\text { Not indicated/treatment } \\
\text { complete }\end{array}$ & $\begin{array}{l}\text { STOP the medication; monitor the outcome after stopping } \\
\text { medication }\end{array}$ & $\begin{array}{l}\text { Patient at advanced age on statin; } \\
\text { antibiotics after treatment completed }\end{array}$ \\
\hline$E$ & $\begin{array}{l}\text { Every medication has an } \\
\text { indication }\end{array}$ & $\begin{array}{l}\text { Reassess or justify need for medication; stop, reduce or } \\
\text { change }\end{array}$ & $\begin{array}{l}\text { Omeprazole but heartburn managed } \\
\text { with diet and/or lifestyle changes }\end{array}$ \\
\hline
\end{tabular}

In 2016, the VIONE (Vital, Important, Optional, Not indicated/treatment complete, and Every medication has an indication) deprescribing tool was developed by Saraswathy Battar, MD, at Central Arkansas Veterans Healthcare System (CAVHS) in Little Rock, as a system that could go beyond medication reconciliation (Table 1). Health care providers (HCPs) and pharmacists evaluate each medication that a patient has been prescribed and places each medication in a VIONE category. Prescribers may then take the opportunity to deprescribe or discontinue medications if deemed appropriate based on their clinical assessments and shared decision making. ${ }^{8}$ Traditionally, medication reconciliation involves the process of obtaining a complete and accurate list of medications as reported by a patient or caregiver to a HCP. VIONE encourages HCPs and pharmacists not only to ensure medication lists are accurate, but also that each medication reported is appropriate for continued use. In other words, VIONE is meant to help implement deprescribing at opportune times. More than 14,000 medications have been deprescribed using the VIONE method, resulting in more than $\$ 2,000,000$ of annualized cost avoidance after just 1 year of implementation at CAVHS. ${ }^{9}$

VIONE consists of 2 major components in the Computerized Patient Record System (CPRS): a template and a dropdown discontinuation menu. The template captured patient allergies, pertinent laboratory data, the patient's active problem list and applicable diagnoses, and active medication list. Patient aligned care team (PACT) pharmacists used the information captured in the template to conduct medication reconciliations and polypharmacy reviews. Each medication is categorized in VIONE using data collected during reviews. A menu delineates reasons for discontinuation: optional, dose decrease, no diagnosis, not indicated/treatment complete, discontinue alternate medication prescribed, and patient reported no longer taking. The discontinuation menu allowed PACT pharmacists and physicians to choose 1 VIONE option per medication to clarify the reason for discontinuation. VIONE-based discontinuations are recorded in CPRS and identified as deprescribed.

At the time of this project, $>30$ US Department of Veterans Affairs (VA) facilities had adopted VIONE. Use of VIONE at VA Southern Nevada Healthcare System (VASNHS) in North Las Vegas has been incorporated in the everyday practices of home-based primary care pharmacists and physicians but has yet to be implemented in other areas of the facility. The purpose of this project was to determine the impact of the VIONE tool on polypharmacy and cost avoidance at VASNHS when used by primary care physicians (PCPs) and PACT primary care clinics.

\section{METHODS}

Veterans receiving care at VASNHS aged $\geq 65$ years with $\geq 10$ active medications noted in CPRS were included in this project. PACT pharmacists and physicians were educated on the proper use of the VIONE tool prior to its implementation. Education included a 15-minute slide presentation followed by dissemination of a 1-page VIONE 
TABLE 2 Discontinuation Dropdown Menu Options Selected

\begin{tabular}{lc} 
VIONE Option Selections & Medications, No. \\
\hline Patient reported no longer taking & 613 \\
\hline Not indicated/treatment complete & 235 \\
\hline No diagnosis & 115 \\
\hline Dose decrease & 65 \\
\hline Optional & 32
\end{tabular}

tool handout during a PACT all-staff clinic meeting.

Data were collected for 3 months before and after the intervention. Data were made available for assessment by the Automated Data Processing Application Coordinator (ADPAC) at VASNHS. The ADPAC created and generated an Excel spreadsheet report, which listed all medications deprescribed using the VIONE method. The primary endpoint was the total number of medications discontinued using the VIONE template and/ or discontinuation menu. For the purpose of this project, appropriate discontinuation was considered any prescription deprescribed, excluding medical supplies, by pharmacists and PCPs who received VIONE education.

The secondary endpoint was the estimated annualized cost avoidance for the facility (Figure). The calculation does not include medications discontinued due to the prescription of an alternative medication or dose decreases since these VIONE selections imply that a new prescription or order was placed and the original prescription was not deprescribed. Annualized cost avoidance was determined with use of the VIONE dashboard, a database that retrospectively gathers information regarding patients at risk of polypharmacy, polypharmacy-related ADEs, and cost. Manual adjustments were made to various parameters on the Veterans Integrated Service Network 15 VIONE dashboard by the author in order to obtain data specific to this project. These parameters allowed selection of service sections, specific staff members or the option to include or exclude chronic or nonchronic medications. The annualized cost avoidance figure was then compared to raw data pulled by a VIONE dashboard correspondent to ensure
TABLE 3 Top 5 Medication Classes Deprescribed

\begin{tabular}{lc} 
Medication Classes & Deprescribed, No. \\
\hline Vitamins/supplements & 206 \\
\hline Pain & 164 \\
\hline Antimicrobial & 102 \\
\hline Hypertension & 98 \\
\hline Diabetes mellitus & 68
\end{tabular}

the manual calculation was accurate. Finally, the 5 most common classes of medications deprescribed were identified for information purposes and to provide a better postulation on the types of medications being discontinued using the VIONE method.

\section{RESULTS}

A total of 2,442 veterans met inclusion criteria, and the VIONE method was applied to 598 between late October 2018 and January 2019. The 13 PACT pharmacists contacted at least 10 veterans each, thus at least 130 were randomly selected for telephone calls to perform polypharmacy reviews using the VIONE note template. The discontinuation menu was used if a medication qualified to be deprescribed. After 3 months, 1986 prescriptions were deprescribed using VIONE; however, 1060 prescriptions were considered appropriately deprescribed (Table 2). The 13 PACT pharmacists deprescribed 361 medications, and the 29 PACT physicians deprescribed 699 medications. These prescriptions were then separated into medication categories to determine the most common discontinued classes. Vitamins and supplements were the medication class most frequently deprescribed (19.4\%), followed by pain medications (15.5\%), antimicrobial agents (9.6\%), antihypertensive medications (9.2\%), and diabetes medications (6.4\%) (Table 3). The top 5 medication categories accounted for $60 \%$ of all medications appropriately deprescribed.

The estimated annualized cost avoidance for all medications deprescribed in the 3 -month project period was $\$ 84,030.46$. To provide the most appropriate and accurate calculation, medication classes excluded 
FIGURE Annualized Cost Avoidance Equation

\begin{tabular}{c} 
Annualized Cost Avoidance $=$ \\
$\frac{\text { Price Per Dispensed Unit } \times \text { Quantity Dispensed }}{\text { Days Supply }} \times$ Days of Cost Avoidance Achieved (max. 365) \\
\hline
\end{tabular}

from this figure were acute or short-term prescriptions and antimicrobial agents. Medications prescribed short-term typically are not suitable to continue for an extended period, and antimicrobial agents were excluded since they are normally associated with higher costs, and may overestimate the cost avoidance calculation for the facility.

\section{DISCUSSION}

The outcomes for the primary and secondary endpoints of this project illustrate that using VIONE in PACT primary care clinics had a notable impact on polypharmacy and cost avoidance over a short period. This outcome can be attributed to 2 significant effects of using the deprescribing tool. VIONE's simplicity in application allowed clinicians to incorporate daily use of the tool with minimal effort. Education was all that was required to fully enable clinicians to work together successfully and exercise collaborative practice to promote deprescribing. VIONE also elicited a cascade of favorable effects that improve patient safety and health outcomes. The tool aided in identification of PIM, which helped reduce polypharmacy and medication burden. The risk for DDIs and ADEs may decrease; therefore, the incidence of falls, need for emergency department visits or inpatient care related to polypharmacy may decline. Less complex medication regimens may alleviate issues with adherence and avoid the various consequences of polypharmacy in theory. Simplified regimens can potentially improve disease management and quality of life for patients. Further studies are needed to substantiate deprescribing and its true effect on patient adherence and better health outcomes at this time. ${ }^{10}$

Reducing polypharmacy can lead to cost savings. Based on the results of this 3-month study, we expect that VASNHS would save more than $\$ 84,000$ by reducing polypharmacy among its patients. Those savings can be funneled back into the health care system, and allotted to necessary patient care, prescriptions, and health care facility needs.

\section{Limitations}

There are some important limitations to this study. Definitions of polypharmacy may vary from one health care facility to another. The cutoffs for polypharmacy may differ, causing the prevalence of polypharmacy and potential costs savings to vary. Use of VIONE may be inconsistent among users if not previously educated or properly trained. For instance, VIONE selections are listed in the same menu as the standard CPRS discontinuation options, which may lead to discontinuation of medical supplies or laboratory orders instead of prescriptions.

The method of data analysis and project design used in this study may have been subject to error. For example, the list of PCPs may have been inaccurate or outdated, which would result in an over- or underrepresentation of those who contributed to data collection. Furthermore, there is some volatility in calculating the total cost avoidance. For example, medications for chronic conditions that were only taken on an as needed basis may have overestimated savings. Either under- or overestimations could occur when parameters are adjusted on the VIONE discontinuation dashboard without appropriate guidance. With the ability to manually adjust the dashboard parameters, dissimilarities in calculations may follow.

\section{CONCLUSIONS}

The VIONE tool may be useful in improving patient safety through deprescribing and discontinuing PIM. Decreasing the number of medications being taken concomitantly by a patient and continuing only those that are imperative in their medical treatment is the first step to reducing the incidence of polypharmacy. Consequently, chances of ADEs or DDIs are lessened, especially among older individuals who are considered high risk 
for experiencing the detrimental effects that may ensue. These effects include geriatric-related syndromes, increased risk of fall, hospital visits or admissions, or death. Use of VIONE easily promotes collaboration among clinicians to evaluate medications eligible for discontinuation more regularly. If this deprescribing tool is continuously used, costs avoided can likely be maximized within VA health care systems.

The results of this project should serve as an incentive to push for better prescribing practices and increase deprescribing efforts. It should provoke the need for change in regimens and the subsequent discontinuation of prescriptions that are not considered vital to continue. Finally, the result of this project should substantiate the positive impact a deprescribing tool can possess to avert the issues commonly associated with polypharmacy.

\section{Author disclosures}

The authors report no actual or potential conflicts of interest with regard to this article.

\section{Disclaimer}

The opinions expressed herein are those of the authors and do not necessarily reflect those of Federal Practitioner, Frontline Medical Communications Inc., the US Government, or any of its agencies.

\section{References}

1. Centers for Disease Control and Prevention, National Center for Health Statistics. Health, United States, 2013: with special feature on prescription drugs. Published May 2014. Accessed May 13, 2021. https://www.cdc.gov/nchs/data /hus/hus13.pdf

2. Masnoon N, Shakib S, Kalisch-Ellett L, Caughey GE. What is polypharmacy? A systematic review of definitions. BMC Geriatr. 2017;17(1):230. Published 2017 Oct 10. doi:10.1186/s12877-017-0621-2

3. Parulekar MS, Rogers CK. Polypharmacy and mobility. In: Cifu DX, Lew HL, Oh-Park M., eds Geriatric Rehabilitation. Elsevier; 2018. doi:10.1016/B978-0-323-54454-2.12001-1

4. Rieckert A, Trampisch US, Klaaßen-Mielke R, et al. Polypharmacy in older patients with chronic diseases: a crosssectional analysis of factors associated with excessive polypharmacy. BMC Fam Pract. 2018;19(1):113. Published 2018 Jul 18. doi:10.1186/s12875-018-0795-5

5. Thompson CA. New medication review method cuts veterans' Rx load, saves millions. Am J Health Syst Pharm. 2018;75(8):502-503. doi:10.2146/news 180023

6. Reeve E. Deprescribing tools: a review of the types of tools available to aid deprescribing in clinical practice. J Pharm Pract Res. 2020;50(1):98-107. doi:10.1002/jppr.1626

7. Fried TR, Niehoff KM, Street RL, et al. Effect of the Tool to Reduce Inappropriate Medications on Medication Communication and Deprescribing. J Am Geriatr Soc. 2017;65(10):2265-2271. doi:10.1111/jgs. 15042

8. Battar S, Dickerson KR, Sedgwick C, et al. Understanding principles of high reliability organizations through the eyes of VIONE, a clinical program to improve patient safety by deprescribing potentially inappropriate medications and reducing polypharmacy. Fed Pract. 2019;36(12):564-568.

9. Battar S, Cmelik T, Dickerson K, Scott, M. Experience better health with VIONE a safe medication deprescribing tool [Nonpublic source, not verified]

10. Ulley J, Harrop D, Ali A, et al. Desprescribing interventions and their impact on medication adherence in communitydwelling older adults with polypharmacy: a systematic review. BMC Geriatr. 2019;19(15):1-13. 